\title{
3 Research Square

\section{Clinical outcomes after total pancreatectomy for malignant neoplasms; a single center retrospective study of 26 cases}

Satoshi Hayama ( $\nabla$ s-hayama@par.odn.ne.jp )

IMS Sapporo Digestive Disease Central General Hospital

Yoshiyasu Ambo

Teine Keijinkai Hospital

Shintaro Takeuchi

Teine Keijinkai Hospital

Minoru Takada

Teine Keijinkai Hospital

Fumitaka Nakamura

Teine Keijinkai Hospital

Nobuichi Kashimura

Teine Keijinkai Hospital

\section{Satoshi Hirano}

Hokkaido University Graduate School of Medicine

\section{Research article}

Keywords: Total pancreatectomy, remnant pancreatectomy, multiple pancreatic tumor, IPMN-associated tumor, de novo PDAC

Posted Date: March 16th, 2020

DOI: https://doi.org/10.21203/rs.3.rs-17343/v1

License: (c) (1) This work is licensed under a Creative Commons Attribution 4.0 International License. Read Full License 


\section{Abstract}

Background: Total pancreatectomy (TP) used to be applied for pancreatic cancer to obtain better oncological clearance or to avoid pancreatic fistulae, but indications have changed. The aim of this study was to clarify recent disease indications and outcomes for this procedure.

Methods: A total of 26 patients who underwent one-stage surgery $(n=11)$ or two-stage surgery $(n=15)$ were analyzed retrospectively.

Results: The postoperative morbidity rate was $26.9 \%$, but no postoperative mortality was encountered. Twenty-two patients had multiple diseases, including intraductal papillary mucinous neoplasm (IPMN) associated tumors in 12 and de novo pancreatic ductal carcinoma (PDAC) in 5. Significantly more T3/T4 and stage III/IV tumors were included in the de novo PDAC group than in the IPMN-associated tumor group (T3/T4: $70 \%$ vs. $16.7 \%$, $p=0.002$; stage III/IV: $100 \%$ vs. $33.3 \%, p=0.012$ ). Two-year survival rates were lower in the de novo PDAC group (20\%) than in the IPMN-associated tumor group $(68.8 \%, p=0.041)$.

Conclusions: TP can be performed safely with low mortality and acceptable morbidity. Our study revealed that the most frequent indication for TP was multiple diseases, and the two main cohorts involved different malignancies: IPMN-associated tumors; and de novo PDAC.

\section{Introduction}

After elective total pancreatectomy (TP) was first performed by Eugene in the United States in 1942 [1], some centers asserted the appropriateness of its indication for pancreatic cancer to improve the prognosis through improved oncological clearance [2], as well as to avoid pancreatic fistulae [3]. High local recurrence rates after partial pancreatectomy [4] suggested that pancreatic cancer might develop multicentrically in the pancreatic gland, making TP a logical choice; however, at present it is acknowledged that not all pancreatic cancers are indicated for TP. Moreover, as a result of improvements in surgical techniques, better care for pancreatic fistulae and the reduced postoperative quality of life (QOL) seen with TP without oncological benefit, these earlier indications for TP have largely been ruled out at present.

With regard to postoperative problems, the complete deficiency of endocrine and exocrine function after TP leads to severe fluctuations in glucose levels and intestinal malabsorption. Nowadays, with the establishment of improved long-acting insulin and pancreatic enzymes, the outlooks for postoperative diabetes (so-called brittle diabetes) and malnutrition status have improved. Such improvements in postoperative care have encouraged the use of TP when indicated. Against such a background, grasping the clinical features of diseases that can once again be indicated for treatment with TP is important, even though earlier indications for TP have largely been contradicted.

The classification and investigation of pathologies effectively treated using TP may provide insights into their biological nature. The aim of the present study was therefore to review the underlying diseases, 
operative results, postoperative nutritional status and prognosis after TP based on modern indications.

\section{Methods}

Between December 2001 and August 2016, a total of 26 patients underwent TP involving total remnant pancreatectomy in Teine Keijinkai Hospital. We retrospectively analyzed clinicopathological features including outcomes for these cases. The end date for this study permitted a minimum follow-up of 6 months. Our indications for TP were simple, as follows:

1. Centrally located pancreatic tumor, to achieve negative surgical margins;

2. Multiple or multifocal parenchymal disease; or

3. Remnant pancreatic tumor.

\section{Descriptions of operative procedures}

All TPs were elective operations performed as open procedures by experienced surgeons. The antrum was resected $3 \mathrm{~cm}$ oral to the pyloric ring and gastrojejunostomy was performed by the antecolic route. Choledochojejunostomy was performed using interrupted absorbable monofilament sutures with or without tube drainage, according to duct size.

\section{Pathological examination}

Intraductal papillary mucinous neoplasm (IPMN) was defined as mucin-producing cystic neoplasm with tall, columnar epithelial cells, with or without papillary projections, with extensive involvement of the main pancreatic duct or its side branches [5]. IPMN consisting of high-grade dysplasia based on the highest grade of dysplasia was defined as non-invasive intraductal papillary mucinous carcinoma (N-IPMC). Pancreatic adenocarcinoma in the background of IPMN was classified as invasive IPMC (I-IPMC) or pancreatic ductal carcinoma (PDAC) concomitant with IPMN. A clear transitional zone between carcinoma and adenoma is found in the former, whereas carcinoma is found separate from IPMN in the latter. In addition, IPMN, IPMC and PDAC concomitant with IPMN were defined as IPMN-associated tumors, and PDAC independent of IPMN was classified as de novo PDAC.

\section{Perioperative management}

Total parental nutrition was usually maintained up to 2 days and blood sugar levels were assessed 6 times per day and controlled using a sliding scale insulin therapy. Oral food intake was started on postoperative day 2, and glycemic control was then achieved by intensive insulin therapy with rapid and long-acting insulin analogs when adequate oral feeding was clinically feasible. Long-acting insulin (initial dose: 2-3 units/day) was induced after stable oral food intake. High-dose oral pancreatic enzymes were routinely administered for all patients.

Diabetic education was performed for all patients postoperatively, and patients were usually discharged to home within 2 weeks postoperatively. 
Follow-up

Patients were followed-up in the output department of endocrinology and surgery or gastrointestinology. Disease recurrence was screened by computed tomography and tumor marker levels for malignant disease for at least 5 years. Serum albumin, glycated hemoglobin (HbA1c) and weight were checked on each visit to the outpatient clinic.

Statistical analysis

Statflex version 6 software (Artech Co., Osaka, Japan) was used for all statistical analyses. The ${ }^{2}$ and Fisher's exact test were used for the analysis of categorical variables, and Student's $t$ test was used for the analysis of continuous variables. Values of $p<0.05$ were considered significant.

\section{Results}

Preoperative features are summarized in Table 1 for the 11 patients who underwent one-stage surgery (0TP) and the 15 patients who underwent two-stage surgery (T-TP). The sex ratio different significantly between groups, with women comprising $18.2 \%$ of the O-TP group and $60 \%$ of the T-TP group. No significant differences in median age or median body weight at surgery were seen. Preoperative incidence of diabetes tended to be more frequent in the T-TP group (60\%) than in the 0-TP group (36.4\%), although that difference was not significant. Performance status was 0 in all patients. The reason for O-TP was multiple diseases in 7 patients and extended disease in 4 patients. The breakdown of multiple diseases was PDAC/IPMN (IPMC)in 3 patients and PDAC/PDAC, PDAC/neuroendocrine tumor (NET), multiple acinar cell carcinomas, and multiple renal cell carcinoma (mRCC) metastases in 1 patient each. In the TTP group, diseases leading to initial pancreatectomy and remnant pancreatectomy were IPMN (C) / IPMN (C) in 7 patients, PDAC / PDAC in 4 patients and IPMN / PDAC in 2 patients.

Operative results are shown in Table 2. Planned partial pancreatectomy was converted to TP from intraoperative frozen-section findings in 2 patients from the O-TP group. Ten patients underwent pancreaticoduodenectiomy (PD) and 5 patients underwent distal pancreatectomy (DP) in the T-TP group. In all 26 patients, clear histological margins were found according to plan via TP. Compared with the 0-TP and T-TP groups, the O-TP group showed longer operation time (512 min vs. 417 min, respectively; $p=$ $0.009)$ and more bleeding (729 $\mathrm{mL}$ vs. $300 \mathrm{~mL}$, respectively; $\mathrm{p}=0.082)$ compared with the T-TP group. On the other hand, no significant differences were seen between 0-TP and T-TP groups in terms of postoperative hospital stay (19 days vs. 21 days, respectively) or morbidity rate (27.3\% vs. $26.7 \%$, respectively), and no mortality was seen in either group.

Long-term survival rate is shown in Figure 1. For the 26 TP patients, median follow-up was 21.5 months (range, 6-84 months). Eight patients died of disease recurrence and one died of other causes. Overall 5year survival rate was $64.3 \%$. No significant differences in survival rate were seen between 0-TP and T-TP groups. 
As shown in Table 1, 22 of the 26 patients (84.6\%) underwent TP for multiple diseases, in both O-TP and T-TP groups. Looking at these 22 patients, two major categories of tumor stood out: IPMN-associated tumors (IPMN-associated tumor group; $n=12$ ) and de novo PDAC (de novo PDAC group; $n=5$ ). IPMNassociated tumors included IPMN, IPMC and PDAC concomitant with IPMN. Pathological findings for these patients are compared in Table 3. The 5 patients in the de novo PDAC group had 10 tumors in all, whereas the 12 patients in the IPMN-associated tumor group had 24 tumors. Significantly more T3/T4 and stage III/IV tumors were seen in the de novo PDAC group than in the IPMN-associated tumor group (T3/T4: $70 \%$ vs. $16.7 \%$, respectively; $p=0.002$; stage III/IV: $100 \%$ vs. $33.3 \%$, respectively; $p=0.012$ ).

Long-term survival rate was compared between cohorts (Fig. 2). The 2-year survival rate was significantly lower in the de novo PDAC group $(20 \%)$ than in the IPMN-associated tumor group $(68.8 \% ; p=0.041)$.

Moreover, comparing patients with T-TP between these cohorts revealed the interval from initial surgery tended to be shorter in the de novo PDAC group (26 months) than in the IPMN-associated tumor group (86 months; $p=0.215$ ) (Fig. 3), although this difference was not significant.

Evaluations for nutritional status and glycemic control in the long-term are shown in Table 4. Median insulin requirement and $\mathrm{HbA} 1 \mathrm{c}$ level were $18 \mathrm{U}$ and $8.0 \%$, respectively. No patients died from diabetic complications. Postoperative weight reduction was obvious (median: $-11.6 \%$ ), but serum albumin level was within normal limits. Moreover, all patients were discharged to home and returned to normal or acceptable function in society. No significant difference was seen between 0-TP and T-TP groups in terms of insulin requirement, $\mathrm{HbA} 1 \mathrm{c}$ level, serum albumin level and weight change.

\section{Discussion}

After elective TP was first performed in the United States in 1942 [1], this procedure was applied to improve prognosis for advanced PDAC or prevent pancreatic fistulae after partial pancreatectomy [6], but the results of these attempts were disappointing. As a result, TP with curative intent for pancreatic tumor tends to be an uncommon operation for many surgeons. However, multifocal diseases such as IPMN remains an indication for TP, and considering recent refinements in diagnostic modalities and therapeutic interventions for primary tumors, surgeons seem likely to face patients requiring TP more frequently. Moreover, postoperative diabetes has been better managed by improvements in the rapid and long-acting insulin analogs and their combined use, and malnutrition status due to exocrine deficiency has also become manageable with high-quality pancreatic enzyme formula, resulting in better QOL. In this context, clinical cases that require TP involving remnant pancreatectomy may increase. In fact, in our hospital, 23 of 26 TP (88.4\%) were performed in the second half of the study period, between 2009 and 2016.

Regarding indications for TP, our study revealed that the most frequent indication was multiple diseases; whether synchronous or metachronous diseases, 22 patients $(22 / 26,84.6 \%)$ underwent TP for multiple diseases. The most common multiple disease was IPMN-associated tumor such as IPMN, IPMC or PDAC concomitant with IPMN, as multifocal occurrence of IPMNs has been reported elsewhere [7-9]. In our case series, IPMN-associated tumors show a lower tumor grade, earlier stage at surgery and favorable 
prognosis, suggesting an underlying nature of low malignancy. The opposing cohort of patients with multiple diseases were those with de novo PDACs. De novo PDACs showed a higher tumor grade and more advanced stage at surgery, suggesting these tumors represent more aggressive tumors. The de novo PDAC group must represent one of the main multifocal pancreatic diseases, although fewer patients showed de novo PDACs (5/22, 22.7\%) than IPMN-associated tumors. This lower frequency compared with IPMN-associated tumors could be partially explained by the fact that the prognosis of patients with PDAC after "initial surgery" is poor, and improvement of treatment outcomes for the primary cancer could result in more cases of secondary PDAC representing multifocal disease. In fact, the cumulative 5-year incidence of second primary PDAC has been reported as $17.7 \%$, markedly higher than that of metachronous gastric cancer [10]. Moreover, comparison of patients with T-TP between the de novo PDAC and IPMN-associated tumor cohortsrevealed that the interval from initial surgery was shorter in the de novo PDAC group. These results suggested that surveillance for remnant pancreatic malignancy in the de novo PDAC group should be mandatory from the early period and at shorter intervals, along with monitoring for systemic recurrence. Ultimately, PDAC patients with multifocal potential will probably be identified through new genetic markers in the future, and if so, TP as a prophylactic surgery could achieve complete cure for patients in this group.

Although very few reports have analyzed the survival rate for secondary PDAC, Zhou et al [11] reported pooled analysis for 55 patients with secondary PDAC and the 5-year survival rate was $40.6 \%$, higher than in patients with primary PDAC after initial pancreatectomy [12].

Conversely, the problem in the IPMN-associated tumor group is that both secondary PDAC and IPMC often recur after a considerably long interval, although these patients rarely develop recurrence outside the remnant pancreas, nor did any patients with non-invasive IPMC or IPMN, as reported [5]. In our study, most secondary tumors in the IPMN-associated tumor group were found at an earlier stage, indicating that follow-up screening contributes to early detection for secondary tumors in this group.

In this study, comparative review between the 0-TP and T-TP groups provided little information. One reason was that various diseases were mixed between both groups. With regard to the operative procedure, 0-TP was naturally associated with longer operation time and greater bleeding compared to TTP, although the extent of adhesion was another factor associated with surgical difficultyin some cases in the T-TP group.

Regarding postoperative nutritional status, glycemic control was not as difficult in our study as suggested in the past [13]. In accordance with standard practice in our institution, all TP patients in this study were controlled by intensive insulin therapy in an attempt to mimic the normal pattern of insulin secretion, and to deliver replacement insulin using the concepts of basal and bolus insulin coverage. In addition to intensive insulin therapy, pancreatic enzyme formula in pancreatic enzyme replacement therapy (PERT) was indispensable, preventing fat maldigestion and malabsorption as well as deficiencies of fat-soluble vitamins as a consequence of exocrine pancreatic insufficiency $[14,15]$. Moreover, PERT reduces glycemic 
variability through the prevention of fatty stool [16]. PERT combined with dietary management followed by an increase in insulin requirement can result in improvement of the nutrition status of patients.

The median $\mathrm{HbA} 1 \mathrm{c}$ level of $8.0 \%$ in our patients was slightly high as in other reports [17-19], but at a level at which no life-threatening complications attributable to diabetes or hypoglycemia ordinarily occur [18]. One limitation to our study was that the number of patients was small and some patients had not yet been followed for the long term, because TP remains a relatively rare procedure. Still, this study is valuable in that we focused on two entities of multifocal disease and because few reports have dealt with consecutive cases of TP from the same institution. Accumulation of additional cases is needed to definitively characterize the efficacy and long-term clinical outcomes of this surgical procedure.

\section{Conclusion}

In conclusion, TP is a feasible procedure with low mortality and acceptable morbidity. Our study revealed that the most frequent indication for TP is currently multiple disease, comprising two main cohorts with different malignancy: IPMN-associated tumors; and de novo PDAC. After partial pancreatic resection for pancreatic tumors, care must be taken regarding recurrence in the remnant pancreas. Metachronous recurrence can occur earlier in PDAC patients, but often recurs after a considerably longer interval in patients with IPMN-associated tumors.

\section{Declarations}

\section{Acknowledgements}

Not applicable

\section{Author's contributions}

S Ha, Y A, M T, F N and N K designed the research; S Ha, S T and S Hi analyzed and interpreted the data;

S H wrote the paper; $\mathrm{S} \mathrm{H}$ and S Hi drafted and/or revised the manuscript. All authors read and approved the final manuscript.

\section{Funding}

The authors have no financial ties to disclose. 
Availability of data and materials

All data and materials are contained within the manuscript.

\section{Ethics approval and consent to participate}

This study was approved by the Teine Keijinkai Hospital Ethics Committee.

Written informed consent was obtained from all individual participants included in the study.

\section{Consent for publication}

Not applicable

Competing interests: The authors declare that they have no conflicts of interest.

\section{References}

1. Priestley JT, Comfort MW, Radcliffe J. Total pancreatectomy for hyperinsulinism due to an islet-cell adenoma. Ann Surg. 1944; 119:211-21.

2. Collins JJ Jr, Craighead JE, Brooks JR. Rationale for total pancreatectomy for carcinoma of the pancreatic head. N Engl J Med. 1966; 274:599-602.

3. Barbier L, Jamal W, Dokmak S, Aussilhou B, Corcos O, Ruszniewski P, et Impact of total pancreatectomy: short- and long-term assessment. HPB (Oxford). 2013; 15:882-92.

4. Westerdahl J, Andr?n-Sandberg A, Ihse I. Recurrence of exocrine pancreatic cancer-local or hepatic? Hepatogastroenterology. 1993; 40:384-7.

5. Rezaee N, Barbon C, Zaki A, He J, Salman B, Hruban RH, et al. Intraductal papillary mucinous neoplasm (IPMN) with high-grade dysplasia is a risk factor for the subsequent development of pancreatic ductal adenocarcinoma. HPB (Oxford). 2016; 18:236-46.

6. Suzuki S, Kajiyama H, Takemura A, Shimazaki J, Nishida K, Shimoda M. The Clinical outcomes after total pancreatectomy. 2017; 34:142-50.

7. Fritz S, Schirren M, Klauss M, Bergmann F, Hackert T, Hartwig W et al. Clinicopathologic characteristics of patients with resected multifocal intraductal papillary mucinous neoplasm of the pancreas. Surgery. 2012; 152:S74-80.

8. Cunningham SC, Hruban RH, Schulick RD. Differentiating intraductal papillary mucinous neoplasms from other pancreatic cystic lesions. World J Gastrointest Surg. 2010; 2: 331-6. 
9. Ohtsuka T, Kono H, Tanabe R, Nagayoshi Y, Mori Y, Sadakari Y, et al. Follow-up study after resection of intraductal papillary mucinous neoplasm of the pancreas; special references to the multifocal lesions and development of ductal carcinoma in the remnant pancreas. Am J Surg. 2012; 204:44-8.

10. Ishida J, Toyama H, Matsumoto I, Asari S, Goto T, Terai S, et al. Second primary pancreatic ductal carcinoma in the remnant pancreas after pancreatectomy for pancreatic ductal carcinoma: High cumulative incidence rates at 5 years after pancreatectomy. Pancreatology. 2016;16:615-20.

11. Zhou Y, Song A, Wu L, Si X, Li Y. Second pancreatectomy for recurrent pancreatic ductal adenocarcinoma in the remnant pancreas: A pooled analysis. Pancreatology. 2016; 16:1124-8.

12. Rutter CE, Park HS, Corso CD, Lester-Coll NH, Mancini BR, Yeboa DN, et Addition of radiotherapy to adjuvant chemotherapy is associated with improved overall survival in resected pancreatic adenocarcinoma: An analysis of the National Cancer Data Base. Cancer. 2015; 121:4141-9.

13. Dresler CM, Fortner JG, McDermott K, Bajorunas DR. Metabolic consequences of (regional) total pancreatectomy. Ann Surg. 1991; 214:131-40.

14. Gheorghe C, Seicean A, Saftoiu A, Tantau M, Dumitru E, Jinga M, et al. Romanian guidelines on the diagnosis and treatment of exocrine pancreatic insufficiency. J Gastrointestin Liver Dis. 2015; 24:117-23.

15. Struyvenberg MR, Martin CR, Freedman SD. Practical guide to exocrine pancreatic insufficiency Breaking the myths. BMC Med. 2017; 15:29.

16. Crosby J, Bellin MD, Radosevich DM, Chinnakotla S, Dunn TB, Pruett TL, et Gastrointestinal symptoms before and after total pancreatectomy with Islet autotransplantation: the role of pancreatic enzyme dosing and adherence. Pancreas. 2015; 44: 453-8.

17. Jethwa P, Sodergren M, Lala A, Webber J, Buckels JA, Bramhall SR, et al. Diabetic control after total pancreatectomy. Dig Liver Dis. 2006; 38:415-9.

18. Casadei R, Monari F, Buscemi S, Laterza M, Ricci C, Rega D, et al. Total pancreatectomy: indications, operative technique, and results: a single centre experience and review of literature. Updates Surg. 2010; 62:41-6. Review.

19. Roberts KJ, Blanco G, Webber J, Marudanayagam R, Sutcliffe RP, Muiesan P, et al. How severe is diabetes after total pancreatectomy? A case-matched analysis. HPB (Oxford). 2014; 16:814-21.

\section{Tables}

Due to technical limitations, the tables are only available as a download in the supplemental files section.

\section{Figures}


Figure1. Overall survivals of patients after total pancreatectomy

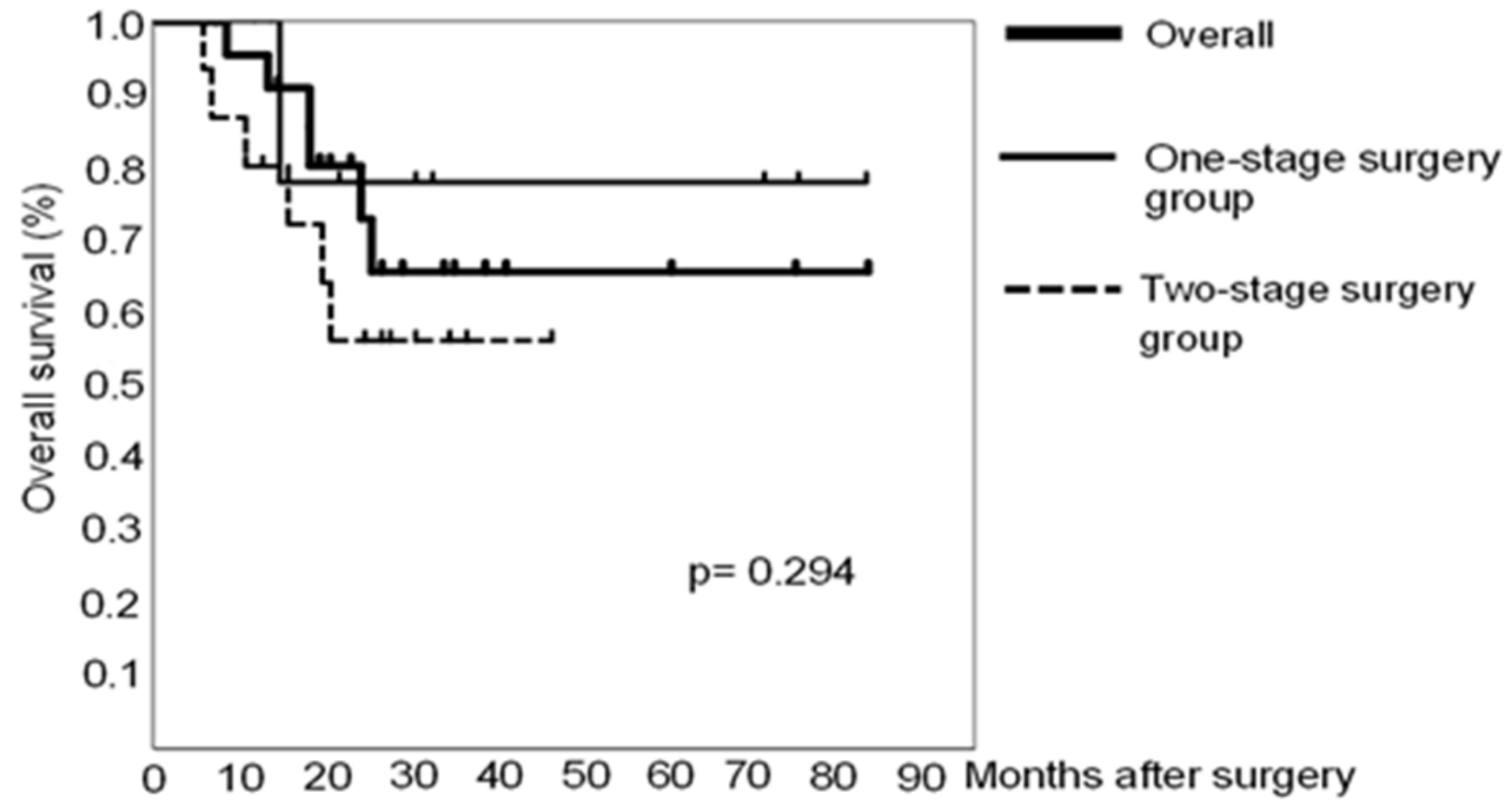

Figure 1

Overall survivals rate of patients after total pancreatectomy. Overall: bold line. One-stage surgery: full line. Two-stage surgery: dashed line.

Figure2. Comparison of IPMN-associated tumor group and de novo PDAC group (overall survival rate)

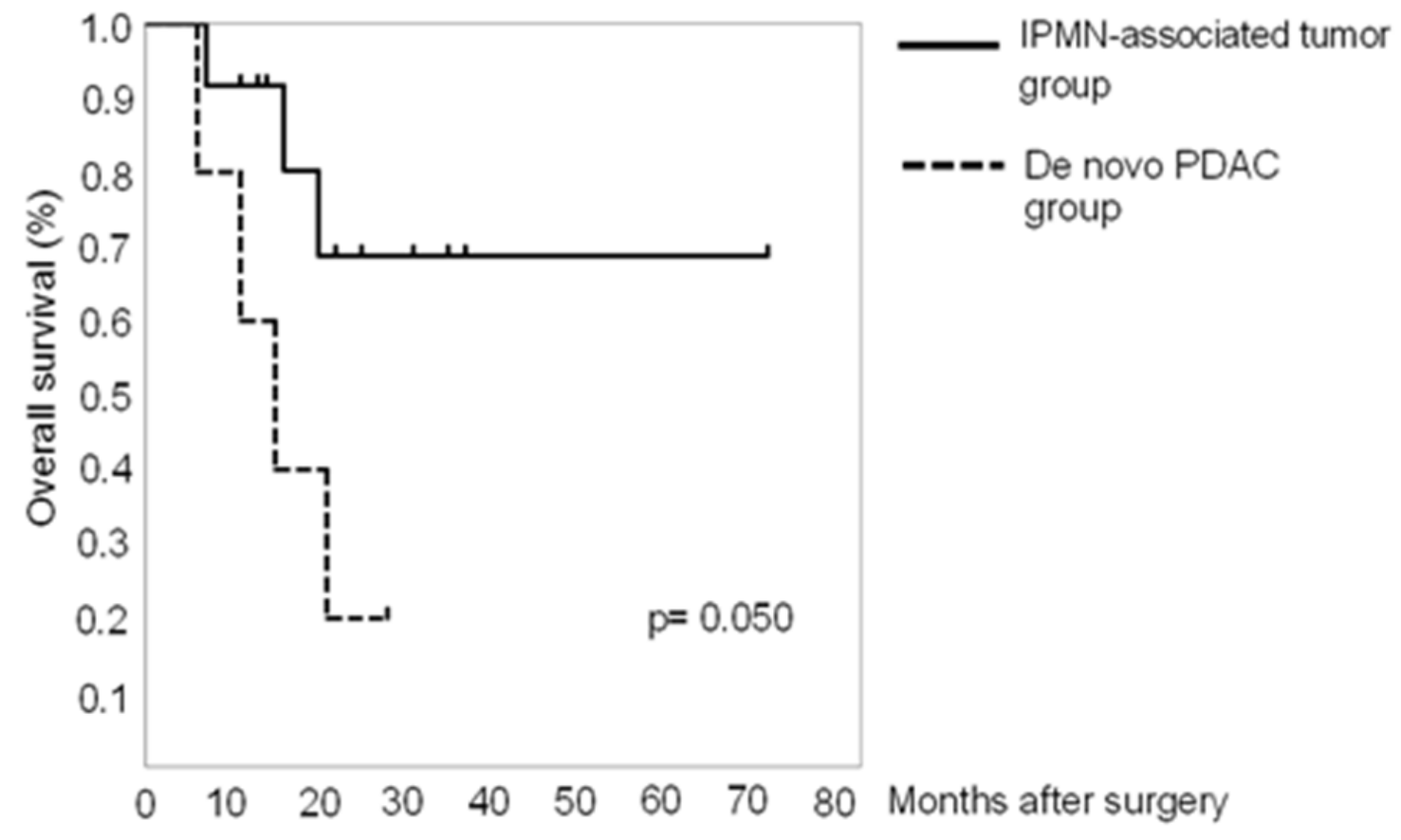


Figure 2

Overall survival rate of IPMN-associated tumor group and de novo PDAC group. IPMN-associated tumor group: full line. De novo PDAC group: dashed line.

Figure3. Comparison of de novo PDAC group and IPMN-associated tumors (interval for second surgery)

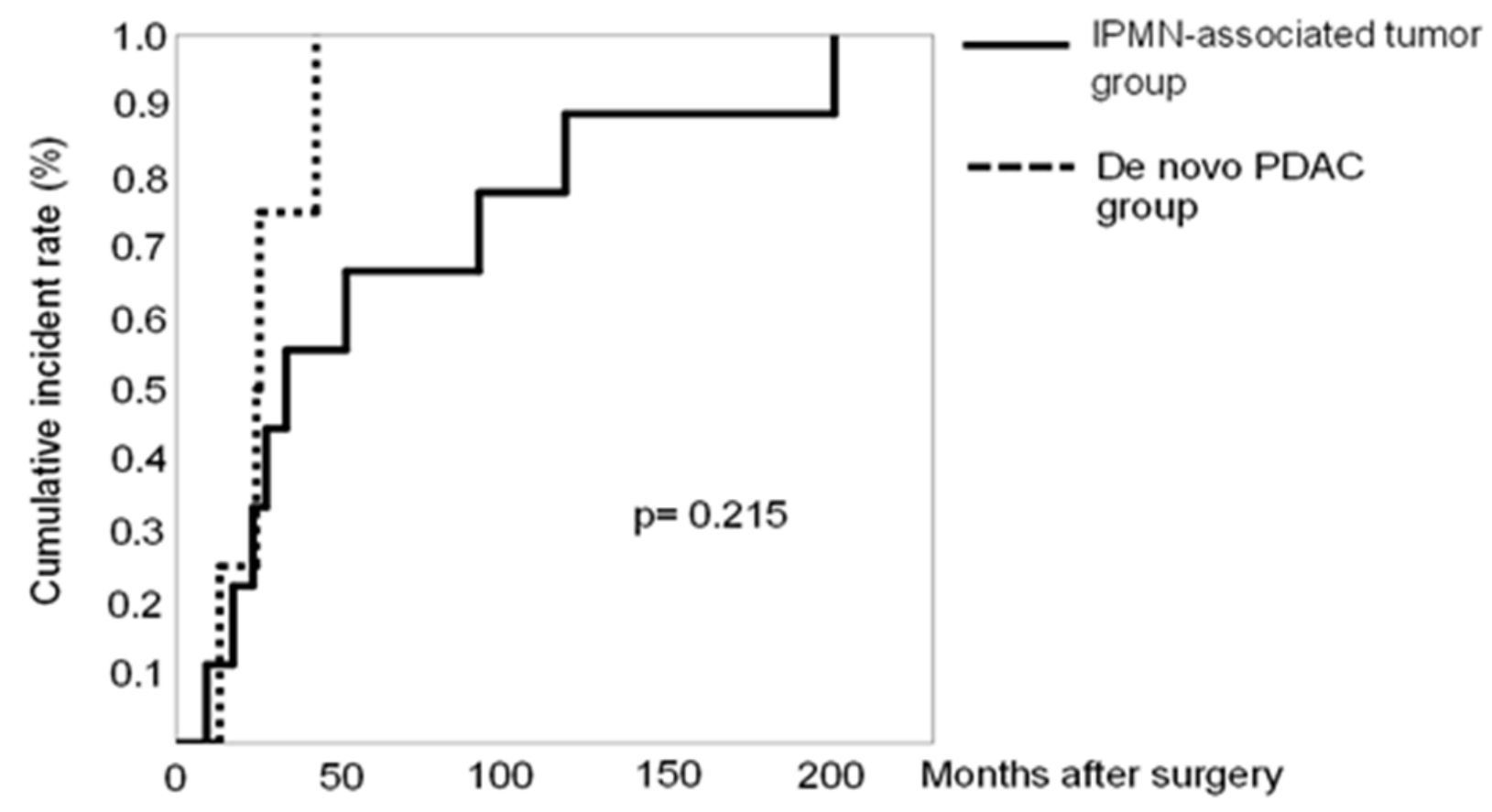

Figure 3

Interval for second surgery. IPMN-associated tumor group: full line. De novo PDAC group: dashed line.

\section{Supplementary Files}

This is a list of supplementary files associated with this preprint. Click to download.

- Tables.pdf 